\title{
Fabio Casale ${ }^{1,2}$, Radames Bionda ${ }^{3}$, Riccardo Falco $^{1}$, Paolo Siccardi ${ }^{1}$, Valentina Toninelli ${ }^{4}$, Diego Rubolini ${ }^{5} \&$ Mattia Brambilla $^{1}$
}

\section{MISURE GESTIONALI IN CAMPO AGRO-PASTORALE PER LA CONSERVAZIONE DELL'AVERLA PICCOLA, LANIUS COLLURIO}

Riassunto - La Provincia del Verbano Cusio Ossola (Piemonte nord-orientale) ha realizzato nel periodo 2003-2007 un Progetto LIFE-Natura incentrato sul sito Natura 2000 "Greto del Toce tra Domodossola e Villadossola". Una delle azioni previste dal progetto attiene la gestione naturalistica di praterie da fieno e di ambienti arbustivi con tecniche che favoriscano specie ornitiche di interesse comunitario, in particolare Lanius collurio. Tali interventi hanno portato a un incremento della popolazione nidificante di tale specie, da 9 a 25 coppie in 5 anni.

Parole chiave - Averla piccola, conservazione, praterie, arbusteti, gestione ambientale, Natura 2000.

Abstract - Habitat management for Red-backed Shrike, Lanius collurio, conservation in farmland systems.

The Province of Verbano Cusio Ossola, North-Eastern Piedmont, carried out a LIFE-Nature project during the period 2003-2007. The project focused on a Natura 2000 site, located along the middle course of river Toce. One of the interventions was directed at the conservation of the breeding population of Lanius collurio through dedicated management of lowland hay meadows (with maintenance of unmown portions) and shrublands (with increasing habitat openness in overgrown shrublands). Those interventions resulted an increase in the local breeding population of the species from 9 to 25 pairs in 5 years.

Key words - Red-backed Shrike, conservation, grasslands, shrubland, habitat management, Natura 2000.

1 Fondazione Lombardia per l'Ambiente, Settore Biodiversità e Aree protette - Piazza Diaz, 7 - I-20123 Milano

2 Università degli Studi dell'Insubria, Unità di Analisi e Gestione Risorse Ambientali - Via Dunant, 3 - I-27100 Varese

3 Società di Scienze Naturali del Verbano Cusio Ossola - Frazione Beola, 18 - I-28868 Baceno (VB)

4 Provincia del Verbano Cusio Ossola, Settore Ambiente ed Energia - Via dell'Industria, 25 I-28924 Verbania

5 Università degli Studi di Milano, Dipartimento di Biologia, Sezione Ecologia - Via Celoria, 20 - I-20133 Milano 


\section{Introduzione}

L'Averla piccola frequenta un'ampia gamma di ambienti semi-aperti posti dal livello del mare sino a $2.000 \mathrm{~m}$, caratterizzati dalla presenza di cespugli (utilizzati come siti di nidificazione), punti sopraelevati (posatoi per la caccia) ed abbondanti popolazioni di insetti (principali prede). Aree ad agricoltura estensiva con mosaico di aree aperte e siepi, coltivazioni arboree, margini boschivi, giovani piantagioni, praterie arbustate, pascoli e radure costituiscono gli ambienti tipici per questa specie (HEAT, 1994; FORNASARI et alii, 1997).

La distruzione ed il deterioramento degli habitat vengono considerati le principali cause del declino dell'Averla piccola in Europa (HеAт, 1994; FORNASARI et alii, 1997). L'incremento della superficie coltivata, l'intensificazione dell'agricoltura e i programmi di riforestazione cui sono state sottoposte vaste aree marginali vengono considerati tra le principali cause di perdita di habitat idonei (JAKOBER \& STAUBer, 1987; Kowalski, 1992). L'intensificazione delle pratiche agricole comporta infatti la scomparsa di aree marginali e siepi, con conseguente incremento dei costi energetici che gli adulti devono sostenere per il reperimento del cibo, costretti a muoversi su aree più vaste (DiEHL, 1971; LUGGER, 1992).

La conservazione dell'Averla piccola in Europa richiede interventi su ampia scala volti al mantenimento degli habitat idonei alla specie, attraverso la promozione dell'agricoltura estensiva, in grado di garantire la presenza di praterie con arbusti sparsi, con una struttura eterogenea dello strato erbaceo ottenuta mediante differenziazione dei tempi e della localizzazione di operazioni di sfalcio e pascolamento (НЕATH, 1994).

Uno studio del 2003, incluso nel Progetto LIFE - Natura denominato "Progetto Integrato LIFE Trebbia", incentrato sui SIC dell'Appennino Piacentino, ha portato all'individuazione delle esigenze ecologiche dell'Averla piccola in aree agricole estensive (BRAMBILLA et alii, 2007), evidenziando come:

1) la densità riproduttiva è influenzata positivamente dalla superficie pascolata / coltivata e dalla superficie occupata da siepi e cespugli;

2) la specie è prevalentemente associata ad aree con disturbo ecologico (utilizzo a scopi agro-pastorali) intermedio, con densità riproduttiva più elevata (5,70 coppie / 10 ha) in aree con pascolo e arbusti sparsi.

Nel quinquennio 2003-2007, la Provincia del Verbano Cusio Ossola ha invece realizzato un Progetto LIFE - Natura dal titolo "Fiume Toce: conservazione di ambienti ripariali a favore dell'avifauna nidificante e migratoria" e relativo al SIC e ZPS "Greto del Toce tra Domodossola e Villadossola".

Tra gli habitat presenti nel sito e oggetto degli interventi gestionali previsti nel progetto rientrano le praterie da fieno e gli arbusteti xerofili a Hippophae rhamnoides, Berberis vulgaris e Crataegus monogyna. 
Il Progetto LIFE prevedeva azioni per il miglioramento degli habitat sopra citati a favore dell'Averla piccola, Lanius collurio, specie inserita nell'Allegato I della Direttiva Uccelli 79/409/CEE. In particolare, in linea con le conoscenze teoriche (vedi sopra), sono state svolte le seguenti tipologie di intervento:

- all'interno delle praterie da fieno: mantenimento di fasce laterali non falciate a rotazione annuale (fino al 15 luglio di ogni anno) di circa 3 metri di ampiezza, come aree di rifugio per invertebrati (anni 20042007);

- negli arbusteti xerofili: controllo tramite taglio a raso con mezzi meccanici di Robinia pseudoacacia, specie altamente invasiva che negli ultimi anni tendeva alla "chiusura" di tali ambienti, e successivo pascolamento e controllo meccanico dei ricacci (anni 2005-2007).

\section{Dati e Metodi}

L'area di studio è costituita dal SIC/ZPS "Greto del Toce da Domodossola a Villadossola”, nelle località idonee alla specie di seguito elencate:

\begin{tabular}{lcc}
\hline Località & Habitat & Superficie (ha) \\
\hline Prati di Vergonte e Prata di Vogogna & Praterie da fieno & 211 \\
Beura - Cardezza & Arbusteti xerofili & 16 \\
\hline Totale area di studio & & $\mathbf{2 2 7}$ \\
\hline
\end{tabular}

Tra metà giugno e fine luglio di ogni anno (2003-2007) le aree ritenute potenzialmente idonee alla specie sono state indagate tramite censimenti effettuati con il metodo del mappaggio (BIBBY \& Burgess, 1997). Ogni area è stata visitata in modo esaustivo tre volte e tutti i contatti ottenuti con individui della specie sono stati riportati in mappe di dettaglio (1:2000).

$\mathrm{Al}$ termine dei sopralluoghi è stata realizzata una carta d'insieme con tutte le osservazioni. L'analisi dei contatti ottenuti, ed in particolare dei contatti simultanei tra individui di territori confinanti, ha consentito di delimitare con buona approssimazione ubicazione e forma dei territori. Le ubicazioni di tutti i territori individuati sono state inserite in ambiente GIS (Geographic Information System), al fine di ottenere una mappa georeferenziata di tutti i dati raccolti. In Tabella I viene riportato il numero di coppie insediatesi nell'area di studio nei 5 anni del progetto.

\section{Conclusioni}

Gli interventi gestionali eseguiti nell'ambito del Progetto LIFE-Natura "Fiume Toce" hanno permesso di mettere in pratica e confermare quanto 
Tab. I - Numero di coppie insediatesi nell'area di studio.

\begin{tabular}{lccccc}
\hline Località & $\mathbf{2 0 0 3}$ & $\mathbf{2 0 0 4}$ & $\mathbf{2 0 0 5}$ & $\mathbf{2 0 0 6}$ & $\mathbf{2 0 0 7}$ \\
\hline Numero coppie in praterie da fieno & 7 & 13 & 16 & 19 & 17 \\
Numero coppie in arbusteti xerofili & 2 & 1 & 4 & 3 & 8 \\
\hline Totale coppie territoriali & $\mathbf{9}$ & $\mathbf{1 4}$ & $\mathbf{2 0}$ & $\mathbf{2 2}$ & $\mathbf{2 5}$ \\
\hline Numero coppie/10 ettari & $\mathbf{0 , 4}$ & $\mathbf{0 , 6}$ & $\mathbf{0 , 9}$ & $\mathbf{1 , 0}$ & $\mathbf{1 , 1}$ \\
\hline
\end{tabular}

emerso in linea teorica dagli studi realizzati nel corso del Progetto LIFENatura "Progetto Integrato Trebbia".

Interventi di gestione attiva di ambienti prativi ed arbustivi hanno infatti permesso di ripristinare condizioni favorevoli alla specie, come attestato dal significativo incremento nel numero di coppie nidificanti, da 9 a 25 in 5 anni, con il raggiungimento di densità più elevate di coppie nidificanti (fino a 5 coppie ogni 10 ettari nell'area ad arbusteti xerofili di Beura-Cardezza, confrontabile con le aree a massima densità dell'Appennino piacentino, Figg. 1-2) in corrispondenza di disturbo ecologico (sfruttamento agricolo-pastorale) intermedio, ottenuto tramite interventi gestionali ('in più') in arbusteto abbandonato e sottrazione all'utilizzo per scopi produttivi ('in meno') in prati da sfalcio in precedenza interamente sfruttati pr la produzione del fieno.

In particolare, dalle esperienze dei due progetti LIFE è emerso quanto segue rispetto alle differenti tipologie gestionali:

\begin{tabular}{|c|c|}
\hline Tipologia gestionale & Risultati \\
\hline Pascolo & $\begin{array}{l}\text { - mantiene l'erba bassa e rada: facilita l'individuazione e } \\
\text { la cattura delle prede } \\
\text { - } \quad \text { rimuove l'accumulo di erba } \\
\text { - } \quad \text { promuove l'abbondanza di potenziali specie-preda }\end{array}$ \\
\hline Sfalcio & $\begin{array}{l}\text { - mantiene l'erba bassa e rada: facilita l'individuazione e } \\
\text { la cattura delle prede } \\
\text { - rimuove l'accumulo di erba }\end{array}$ \\
\hline $\begin{array}{l}\text { Fasce incolte } \\
\text { (non falciate, isole di vegetazione) }\end{array}$ & - promuove l'abbondanza di specie-preda \\
\hline
\end{tabular}

Da ciò ne consegue che le condizioni ideali per l'Averla piccola siano un mosaico di:

- aree pascolate o sfalciate, che consentono l'individuazione e la cattura delle prede;

- aree non coltivate/pascolate, che promuovono l'abbondanza di speciepreda;

- siepi ed arbusti, che favoriscono la disponibilità di posatoi e siti per la nidificazione. 


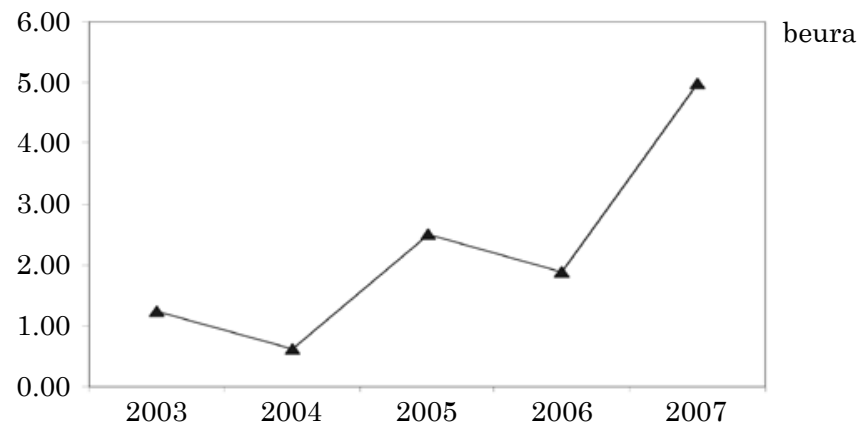

Fig. 1 - Coppie nidificanti/10 ettari in arbusteti xerofili.

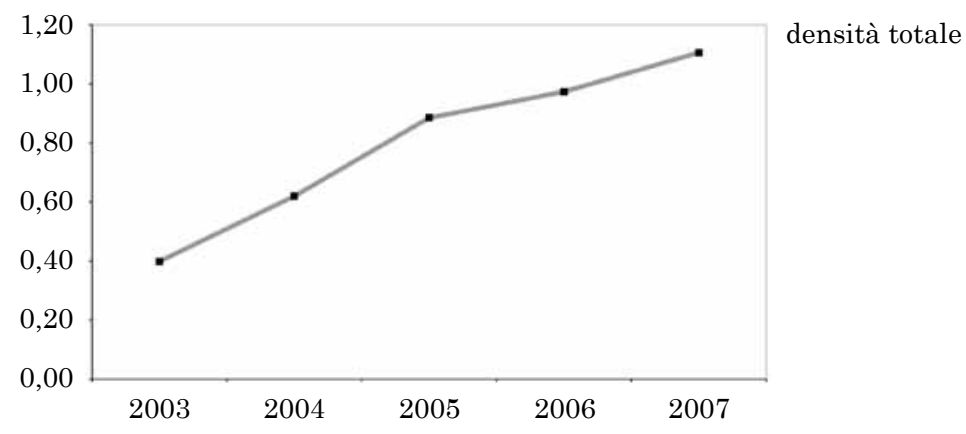

Fig. 2 - Coppie nidificanti/10 ettari: trend complessivo.

\section{BIBLIOGRAFIA}

Bıвву C.J., 1973 - The Red-backed Shrike: a vanishing British species - Bird Study, 20: 103-110. Bibby C.J. \& Burgess N.D., 1997 - Bird Census Techniques - University Press, Cambridge.

Brambilla M., Rubolini D. \& Guidali F., 2007 - Between land abandonment and agricultural intensification: habitat preferences of Red-backed Shrikes Lanius collurio in low-intensity farming conditions - Bird Study, 54: 160-167.

Dienl B., 1971 - Energy requirements of nestling fledgling Red-backed Shrike - Eko polska, 19: $235-248$

Fornasari L., Kurlavicius P. \& Massa R., 1997 - Lanius collurio Red-backed Shrike. In: HaGeMEIJER E.J.M. \& Blair M.J. (eds). The EBCC Atlas of European Breeding Birds: Their Distribution and Abundance - T\& AD Poyser, London, pp. 660-661.

Heath M., 1994 - Red backed Shrike Lanius collurio. In: Tucker, G.M. \& Heath M.F. - Birds in Europe: their conservation status - Cambridge, U.K., BirdLife International, BirdLife Conservation Series no. 3, pp. 410-411.

JAKober H. \& STAUber W., 1987 - Habitat requirements of the Red-backed Shrike and conservation measures - Beih. Veroff. Naturschutz Landschaftspflege, 48: 25-53.

KowALski H., 1992 - Population numbers of Shrike species in Germany: causes of decline, status, conservation strategies - J. Orn., 133: 321-322.

LEFRANC N., 1973 - Notes on the recent history of the Red-backed Shrike Lanius collurio in western Europe - Alauda, 3: 239-252.

LugGer U., 1992 - The influence of the habitat quality on the breeding progress of the Red-backed Shrike and related energetic costs in the adults - J. Orn., 133: 322. 